Article

\title{
A Study on Tissue-Specific Metabolite Variations in Polygonum cuspidatum by High-Resolution Mass Spectrometry-Based Metabolic Profiling
}

\author{
Zhijun Wu ${ }^{1,2}$, Xiaowei Wang ${ }^{1}$, Mo Chen ${ }^{1}$, Hongyan Hu ${ }^{1}$, Jie Cao ${ }^{1}$, Tuanyao Chai ${ }^{1,3, *}$ and \\ Hong Wang $1, * \mathbb{D}$ \\ 1 College of Life Sciences, University of Chinese Academy of Sciences, Yuquan Road, Beijing 100049, China; \\ wuzhijun14b@mails.ucas.ac.cn (Z.W.); wangxiaowei15@mails.ucas.ac.cn (X.W.); \\ chenmo215@mails.ucas.ac.cn (M.C.); huhongyan16@mails.ucas.ac.cn (H.H.); caojie17@mails.ucas.ac.cn (J.C.) \\ 2 School of Life sciences and Biotechnology, Heilongjiang Bayi Agricultural University, Daqing 163319, China \\ 3 Institute of Genetics and Developmental Biology, Chinese Academy of Sciences, Beichen west Road, \\ Beijing 100101, China \\ * Correspondence: tychai@ucas.ac.cn (T.C.); hwang@ucas.ac.cn (H.W.); Tel.: +86-1069672628 (H.W.); \\ Fax: +86-1069672628 (H.W.)
}

Received: 24 February 2019; Accepted: 14 March 2019; Published: 18 March 2019

\begin{abstract}
Polygonum cuspidatum Sieb. et Zucc. is a traditional Chinese herbal medicine widely used to treat tussis, hepatitis and arthralgia. This study identified and quantitatively described the bioactive compounds in different P. cuspidatum tissues. Metabolic profiles of root, stem, leaf, flower, rhizome and seed were determined using high-resolution mass spectrometry in combination with multivariate analyses. In total, 53 metabolites, 8 reported for the first time in this species, were putatively identified and classified mainly as stilbenes, anthraquinones and flavonoids. A principal component analysis, cluster analysis and heatmap were used to depict the correlations between specimens and the relative abundance levels of these compounds in different plant tissues. An orthogonal partial least square discriminant analysis found that 13 metabolites showed distinct differences among the six plant tissues, making them potential discriminative tissue-identification markers. This study will provide guidance in comparing, selecting and exploiting the medicinal uses of different P. cuspidatum tissues.
\end{abstract}

Keywords: Polygonum cuspidatum; LC-MS; metabolomics; multivariate analysis

\section{Introduction}

Polygonum cuspidatum Sieb. et Zucc. is an herbaceous perennial plant of the Polygonaceae family, whose members contain high levels of resveratrol and emodin [1]. The root and rhizome of P. cuspidatum have been widely used to treat tussis, hepatitis, jaundice and arthralgia for thousands of years in China and East Asia [2,3]. P. cuspidatum's pharmacological effects result from the presence of large amounts of phenolic compounds, including anthraquinones, stilbenes, flavonoids and isoflavonoids [4]. Phenolic compounds have antioxidant, anti-microbial and anti-carcinogenic characteristics, which are beneficial to human health $[5,6]$. In addition, the average levels of resveratrol and emodin in P. cuspidatum are much greater than in other plants; therefore, they are used as the indicator compounds to characterize the quality of this plant in Chinese Pharmacopoeia. Thus, P. cuspidatum is considered the main source of natural resveratrol and emodin [7,8].

According to Chinese Pharmacopoeia, the root and rhizome of P. cuspidatum are the main tissues used to make compounds classified as 'Traditional Chinese Medicines' in clinical trials [9], while most of the leaves, flowers, stems and other tissues are usually thrown away. The young tender leaves and stems of P. cuspidatum are edible and used to treat hepatitis and enteritis by the Yi and Miao ethnic 
groups in China $[10,11]$. The phytochemistry and pharmaceutical effects of the root and rhizome of P. cuspidatum have been studied [12,13]; however, less is known about the bioactive composition and medicinal values of the other tissues [14]. In addition, the metabolic profiles and chemical differences among different tissues of P. cuspidatum have not been elucidated systematically.

Metabolomics, especially based on liquid chromatography coupled with high-resolution mass spectrometry (LC-MS), has been widely used to investigate the comprehensive profiles of natural products in herbal plants. Ding et al. [15] established a metabolomics approach based on high-resolution LC-MS and chemometric methods for the quality evaluation and discrimination of natural products. They successfully screened, identified and quantified five markers for the precise quality evaluation of crude and carbonized Pollen Typhae. Geng et al. [16] developed a powerful strategy based on high-resolution quadrupole time-of-flight (Q-TOF) MS to explore the chemical ingredients and transformation mechanisms in cultivated Bulbus Fritillariae cirrhosae. They identified four alkaloid compounds that could be used as potential chemical markers for the classification of cultivated Bulbus Fritillariae cirrhosae samples at different growth stages.

Previously, it was reported that the root and rhizome of P. cuspidatum are the main tissues that have pharmacological effects. We suspect some compounds in other tissues may also be beneficial to human health and could be used in clinical trials. In the present study, metabolic profiles of six different tissues; root, stem, leaf, flower, rhizome and seed of P. cuspidatum, were determined using high-resolution MS. The bioactive compounds of P. cuspidatum were identified, and the relative contents of these compounds among tissues were measured. Statistical analyses confirmed that tissues of $P$. cuspidatum could be distinguished by chemical markers. The results obtained in this study provide valuable insights into the distribution of bioactive compounds in different $P$. cuspidatum tissues and may also provide some practical guidance for choosing the appropriate medicinal tissues for clinical applications.

\section{Results and Discussion}

\subsection{Optimization of the Extraction Procedure and MS Conditions}

The metabolite extraction procedures are crucial for further metabolomics studies. To extract and detect as many compounds as possible, the extraction parameters, including extraction solvents, extraction time, extraction temperature and extraction repetitions, were optimized. The optimized method is described in Section 2.2. The chromatographic conditions and MS parameters were also optimized. Acetonitrile and water, each supplemented with $0.1 \%$ formic acid, were used as mobile phases. The applied gradient was optimized and is described in Section 2.3. Both positive and negative ion modes were performed to determine the suitable ion mode. In negative ion mode, more peaks were detected than in positive ion mode. In addition, a greater sensitivity and clearer structural information were obtained in negative ion mode than in positive ion mode. Therefore, the MS analysis of extracted metabolites was performed in negative ion mode.

\subsection{Comparison of Metabolic Profiles of Different Tissues}

High-resolution Q-TOF MS is an analytical platform that has excellent reproducibility and stability, and is widely used for the identification of metabolites [17]. In the present study, high-resolution LC-Q-TOF MS was used to determine the relative levels and global distribution profiles of metabolites extracted from different tissues of P. cuspidatum. The significant differences and chemical markers among tissues were also investigated. Each group of tissue contained six biological replicates, except the rhizome group had five replicates. The total ion chromatograms of each sample group were very similar. XCMS, a metabolomic data processing platform, was used to analyze the raw data, and 4309 aligned features (including unidentified compounds) were detected. Typical LC-MS spectra are shown in Supplemental Figure S1. 


\subsection{Construction of Predictive Models for the PCA}

Principal component analysis (PCA) is an unsupervised multivariate analysis method for the reduction of complex dataset dimensionality to provide important insights into variations among groups. In the present study, 4309 aligned features were analyzed using a PCA to display the differences among groups. The score plots of the PCA revealed that $31.53 \%$ and $17.1 \%$ of the variation were explained by PC1 and PC2, respectively. The fitness $\left(\mathrm{R}^{2}\right)$ and prediction power $\left(\mathrm{Q}^{2}\right)$ of this PCA model were 0.741 and 0.621 , respectively. The PCA revealed an obvious separation among different tissues, except the root and rhizome groups. Thus, the metabolic constituents of leaves, stems, flowers, seeds, roots and rhizomes differed significantly. The root and rhizome groups clustered together and could not be discriminated through the PCA. Because the root and rhizome have grown underground for many years and accumulated lots of secondary metabolites, their metabolites may differ largely from those of other tissues, and thus, can be discriminated by PC1 (Figure 1). The flower and stem groups were relatively closer than those of other tissues. The chemical profile of the seed group was far from those of other tissues and could be separated by PC2. To determine correlations among the different tissues, the 4309 ion signals detected in this experiment were used in a hierarchical cluster analysis (Figure 1). The PCA and hierarchical cluster analysis showed that samples of different tissues could be discriminated on the basis of LC-MS metabolomics.

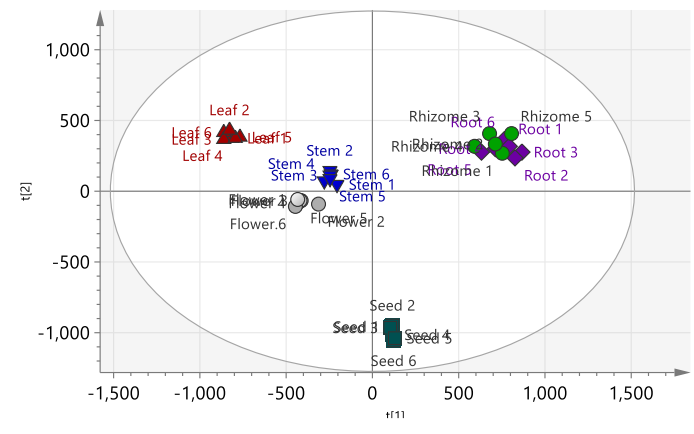

(a)

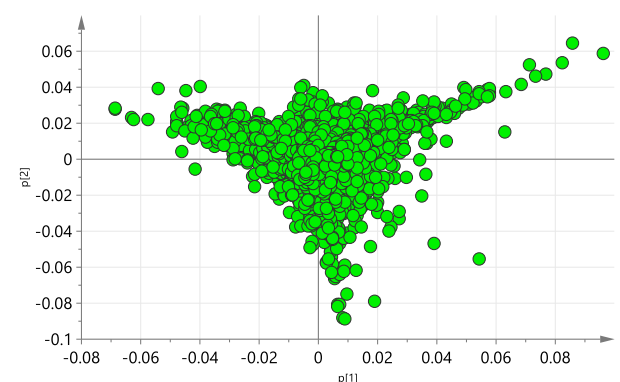

(b)

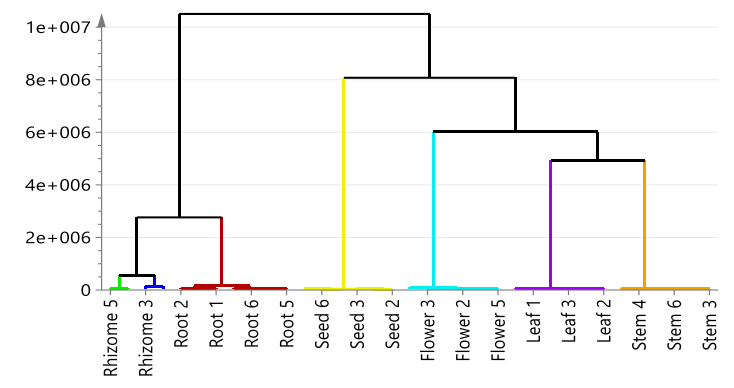

(c)

Figure 1. Principal component snalysis (PCA) and hierarchical cluster analysis on root, rhizome, leaf, flower, stem and seed groups. (a) Scores plot, (b) Loading plot, (c) Hierarchical cluster analysis of all samples. PCA and Hierarchical cluster analysis using all of the 4309 ion signals. Scores plot and loading plot of PCA model gives 2D visual information about the variations among different groups. Unsupervised PCA score plots showing the discrimination of samples. $\mathrm{R}^{2}=0.741, \mathrm{Q}^{2}=0.621$.

\subsection{Tentative Identification of Bioactive Metabolites in P. cuspidatum}

The objective of this study was to identify the major bioactive compounds of P. cuspidatum and potential chemical markers that contribute to significant classification differences among tissues. These compounds were identified by comparing their accurate molecular weights, retention times and fragment patterns with those reported in the literature, databases, such as HMDB, METLIN and MassBank, and reference standards, respectively. We first searched the molecular ion's $m / z$ values 
of compounds in P. cuspidatum found in KNApSAcK, BATMAN-TCM and other references [1-3]. Then, the notably different molecular ions were tentatively identified. The diagnostic ion-filtering strategy for rapid identification and the data mining method were also used based on the methods of Liu et al. and Chang et al. [18,19]. In total, 53 compounds, including stilbenes, anthraquinones, flavonoids, isoflavonoids and other compounds, were annotated from the six tissues. The retention times, molecular ions, classes, MS/MS fragment ions, standard compounds and references for these metabolites are listed in Table 1. Polar compounds were eluted first and the relative nonpolar compounds were eluted last. This trend was in accordance with the elution profile of reverse-phase chromatography [20]. 
Table 1. Characteristic fragment ions, retention times and $m / z$ of metabolites tentatively identified based on reference standard, published literature, METLIN or HMDB database.

\begin{tabular}{|c|c|c|c|c|c|c|c|c|}
\hline Identification & $\begin{array}{l}\text { Precursor } \\
\text { Ion }\end{array}$ & $\begin{array}{l}\text { Molecular } \\
\text { Formula }\end{array}$ & $\begin{array}{l}\text { Predicted } \\
\mathrm{m} / \mathrm{z}\end{array}$ & $\begin{array}{l}\text { Measured } \\
\mathrm{m} / \mathrm{z}\end{array}$ & PPM & RT & ms2 Fragment Ions at Negative Mode & References \\
\hline Tartaric acid & {$[\mathrm{M}-\mathrm{H}]^{-}$} & $\mathrm{C}_{4} \mathrm{H}_{5} \mathrm{O}_{6}$ & 149.0086 & 149.0082 & 2.68 & 0.72 & $\begin{array}{c}\text { 130.9882(2.3), } 105.0215(5.60), 103.0071(1.41), 87.0133(36.37), \\
72.9965(100)\end{array}$ & HMDB, METLIN \\
\hline Malic acid & {$[\mathrm{M}-\mathrm{H}]^{-}$} & $\mathrm{C}_{4} \mathrm{H}_{5} \mathrm{O}_{5}$ & 133.0137 & 133.0133 & 3.01 & 0.72 & $\begin{array}{c}115.0076(19.99), 89.0280(7.69), 72.9970(37.65) \\
71.0176(100), 59.0163(7.95)\end{array}$ & HMDB, METLIN \\
\hline Citric acid & {$[\mathrm{M}-\mathrm{H}]^{-}$} & $\mathrm{C}_{6} \mathrm{H}_{7} \mathrm{O}_{7}$ & 191.0192 & 191.0201 & 4.71 & 0.80 & $\begin{array}{c}173.0125(3.75), 111.0125(100), 87.0126(65.85), 85.0336(76.01) \\
67.0217(21.87), 57.0419(17.03) 103.0106\end{array}$ & HMDB, METLIN \\
\hline Gallic acid & {$[\mathrm{M}-\mathrm{H}]^{-}$} & $\mathrm{C}_{7} \mathrm{H}_{5} \mathrm{O}_{5}$ & 169.0215 & 169.0218 & 1.77 & 0.97 & $\begin{array}{c}\text { 125.0284(100), } 107.0178(8.69), 111.0112(3.30), 97.0326(14.68), \\
79.0236(23.64), 69.0375(19.23)\end{array}$ & $\begin{array}{l}\text { Standard, } \\
\text { METLIN }\end{array}$ \\
\hline $\begin{array}{l}\text { 6-Galloylglucose/ } \\
\text { 2-Galloylglucose }\end{array}$ & {$[\mathrm{M}-\mathrm{H}]^{-}$} & $\mathrm{C}_{13} \mathrm{H}_{15} \mathrm{O}_{10}$ & 331.0663 & 331.0664 & 0.30 & 1.14 & $\begin{array}{c}241.0128(3.41), 211.0290(13.62), 169.0172(100), 151.0054(48.81), \\
137.0258(4.43), 125.0263(43.17), 111.0117(17.48)\end{array}$ & HMDB, [2] \\
\hline $\begin{array}{l}\text { Salicylic acid } \\
\text { beta-D-glucoside }\end{array}$ & {$[\mathrm{M}-\mathrm{H}]^{-}$} & $\mathrm{C}_{13} \mathrm{H}_{15} \mathrm{O}_{8}$ & 299.0772 & 299.0775 & 1.00 & 1.34 & $\begin{array}{c}225.0779(7.62), 179.0422(46.39), 137.0294(100), 119.0522(8.48), \\
93.0370(10.73), 73.0299(23.70), 71.0166(26.89)\end{array}$ & HMDB, METLIN \\
\hline Epiafzelechin 3-gallate & {$[\mathrm{M}-\mathrm{H}]^{-}$} & $\mathrm{C}_{22} \mathrm{H}_{17} \mathrm{O}_{9}$ & 425.0873 & 425.0861 & 2.82 & 2.23 & $\begin{array}{c}\text { 407.0737(100), 255.0812(4.98), } 151.0423(36.55), 137.0292(64.75), \\
125.0224(11.75)\end{array}$ & HMDB \\
\hline $\begin{array}{l}\text { Epigallocatechin/ } \\
\text { Gallocatechin }\end{array}$ & {$[\mathrm{M}-\mathrm{H}]^{-}$} & $\mathrm{C}_{15} \mathrm{H}_{13} \mathrm{O}_{7}$ & 305.0661 & 305.0672 & 3.61 & 2.31 & $\begin{array}{c}219.0648(56.12), 191.0689(34.03), 167.0307(52.37), 139.0480(29.64), \\
137.0315(30.43), 125.0235(100), 111.0429(30.40)\end{array}$ & HMDB, METLIN \\
\hline Glucocaffeic acid & {$[\mathrm{M}-\mathrm{H}]^{-}$} & $\mathrm{C}_{15} \mathrm{H}_{17} \mathrm{O}_{9}$ & 341.0873 & 341.0886 & 3.81 & 2.59 & $\begin{array}{c}221.0438,179.0379(100), 161.0244(6.52), 151.0395(11.84), \\
135.0485(33.41)\end{array}$ & HMDB \\
\hline Catechin & {$[\mathrm{M}-\mathrm{H}]^{-}$} & $\mathrm{C}_{15} \mathrm{H}_{13} \mathrm{O}_{6}$ & 289.0712 & 289.0712 & 0.00 & 2.64 & $\begin{array}{c}271.0601(5.61), 245.0827(23.84), 203.0720(53.62), 151.0424(28.46) \\
109.0320(100)\end{array}$ & $\begin{array}{c}\text { HMDB, } \\
\text { Standard, }[15,21]\end{array}$ \\
\hline Chlorogenic acid & {$[\mathrm{M}-\mathrm{H}]^{-}$} & $\mathrm{C}_{16} \mathrm{H}_{17} \mathrm{O}_{9}$ & 353.0852 & 353.0845 & 1.98 & 2.83 & 191.0585(100), 179.0378(5.08), 161.0270(2.69), 87.0068(2.1) & $\begin{array}{c}\text { HMDB, } \\
\text { METLIN, [19] }\end{array}$ \\
\hline Epigallate catechin gallate & {$[\mathrm{M}-\mathrm{H}]^{-}$} & $\mathrm{C}_{22} \mathrm{H}_{17} \mathrm{O}_{11}$ & 457.0771 & 457.0759 & 2.63 & 2.93 & $\begin{array}{c}\text { 303.0573(100), 287.0546(15.4), } 169.0170(8.76), 165.0557(12.6) \\
161.0138(3.66), 151.0047(75.84), 125.0271(32.68)\end{array}$ & HMDB, METLIN, \\
\hline 3-p-Coumaroylquinic acid & {$[\mathrm{M}-\mathrm{H}]^{-}$} & $\mathrm{C}_{16} \mathrm{H}_{17} \mathrm{O}_{8}$ & 337.0923 & 337.0931 & 2.37 & 3.29 & $\begin{array}{c}277.0685(10.43), 231.0627(2.99), 191.0540(29.44), 173.0443(100), \\
163.0440(26.17), 119.0509(13.82)\end{array}$ & HMDB \\
\hline Epicatechin & {$[\mathrm{M}-\mathrm{H}]^{-}$} & $\mathrm{C}_{15} \mathrm{H}_{13} \mathrm{O}_{6}$ & 289.0712 & 289.0707 & 1.73 & 3.59 & $\begin{array}{c}289.0729(53.01), 245.0939(33.89), 221.0838(18.71), 203.0728(100) \\
151.0438(45.34), 123.0561(73.41), 109.0356(66.28)\end{array}$ & HMDB, $[2,21]$ \\
\hline $\begin{array}{l}\text { Apigenin-6-glucoside } \\
\text { 8-carabinoside }\end{array}$ & {$[\mathrm{M}-\mathrm{H}]^{-}$} & $\mathrm{C}_{26} \mathrm{H}_{27} \mathrm{O}_{14}$ & 563.1395 & 563.1385 & 1.77 & 4.12 & $\begin{array}{c}\text { 563.1375(100), 545.1283(3.52), 503.1142(7.92), 473.1088(10.53), } \\
\text { 443.0946(12.11), 383.0897(10.82), 353.0642(10.79) }\end{array}$ & HMDB, [22] \\
\hline Procyanidin B1 & {$[\mathrm{M}-\mathrm{H}]^{-}$} & $\mathrm{C}_{30} \mathrm{H}_{25} \mathrm{O}_{12}$ & 577.1346 & 577.1371 & 4.33 & 4.36 & $\begin{array}{c}559.1370,535.1225(23.27), 425.0878(38.95), 289.0731(100), \\
125.0276(54.62)\end{array}$ & HMDB, $[2,21]$ \\
\hline 3-galloylprocyanidin B1/B2 & {$[\mathrm{M}-\mathrm{H}]^{-}$} & $\mathrm{C}_{37} \mathrm{H}_{29} \mathrm{O}_{16}$ & 729.1456 & 729.1429 & 3.70 & 4.42 & $\begin{array}{c}\text { 603.1210(10.82), 577.1231(32.4), 441.0865(19.36), 407.0865(100), } \\
289.0757(40.38), 271.0661(8.43), 125.0244(18.96)\end{array}$ & HMDB, METLIN \\
\hline
\end{tabular}


Table 1. Cont

\begin{tabular}{|c|c|c|c|c|c|c|c|c|}
\hline Identification & $\begin{array}{l}\text { Precursor } \\
\text { Ion }\end{array}$ & $\begin{array}{l}\text { Molecular } \\
\text { Formula }\end{array}$ & $\begin{array}{l}\text { Predicted } \\
\quad m / z\end{array}$ & $\begin{array}{l}\text { Measured } \\
\mathrm{m} / \mathrm{z}\end{array}$ & PPM & RT & ms2 Fragment Ions at Negative Mode & References \\
\hline $\begin{array}{l}\text { Resveratrol-4'-o-D- } \\
\text { glucopyranoside }\end{array}$ & $\begin{array}{c}{[\mathrm{M}+} \\
\mathrm{COOH}]-\end{array}$ & $\mathrm{C}_{21} \mathrm{H}_{23} \mathrm{O}_{10}$ & 435.1286 & 435.1290 & 0.92 & 4.67 & $389.1263(2.05), 227.0733(100), 185.0636(1.17), 143.0524(0.64)$ & {$[2,21]$} \\
\hline Epicatechin gallate & {$[\mathrm{M}-\mathrm{H}]^{-}$} & $\mathrm{C}_{22} \mathrm{H}_{17} \mathrm{O}_{11}$ & 441.0822 & 441.0809 & 2.95 & 4.69 & $\begin{array}{c}303.0592(2.11), 290.0796(14.52), 289.0770(47.78), 271.0629(7.1), \\
245.0826(8.03), 169.0182(100), 151.0501(2.87), 125.0281(26.39)\end{array}$ & HMDB, [21-23] \\
\hline Piceid & $\begin{array}{c}{[\mathrm{M}-\mathrm{H}]^{-}} \\
{[\mathrm{M}+} \\
\mathrm{HCOO}]_{-}^{-}\end{array}$ & $\begin{array}{l}\mathrm{C}_{20} \mathrm{H}_{21} \mathrm{O}_{8} \\
\mathrm{C}_{21} \mathrm{H}_{23} \mathrm{O}_{10}\end{array}$ & $\begin{array}{l}389.1240 \\
435.1286\end{array}$ & $\begin{array}{l}389.1236 \\
435.1279\end{array}$ & $\begin{array}{l}1.03 \\
1.61\end{array}$ & $\begin{array}{l}5.09 \\
5.09\end{array}$ & $\begin{array}{l}227.0723(100), 185.0687(5.44), 143.0526(1.28) \\
389.1263(2.05), 227.0733(100)\end{array}$ & $\begin{array}{l}\text { HMDB, METLIN, } \\
\text { Standard, [21] }\end{array}$ \\
\hline Rutin & {$[\mathrm{M}-\mathrm{H}]^{-}$} & $\mathrm{C}_{27} \mathrm{H}_{29} \mathrm{O}_{16}$ & 609.1456 & 609.1480 & 3.94 & 5.09 & $\begin{array}{c}\text { 609.1493(100), 343.0538(1.34), 301.0387(16.57), 300.0319(25.44), } \\
\text { 271.0245(1.43), 255.0305(0.78), 151.0107(0.63), }\end{array}$ & $\begin{array}{l}\text { HMDB, } \\
\text { METLIN, [23] }\end{array}$ \\
\hline Catechin gallate & {$[\mathrm{M}-\mathrm{H}]^{-}$} & $\mathrm{C}_{22} \mathrm{H}_{17} \mathrm{O}_{10}$ & 441.0822 & 441.0809 & 2.95 & 5.23 & $\begin{array}{c}\text { 303.0549(1.63), 289.0750(53.51), 245.0450(13.48), 205.0571(3.22), } \\
169.0179(100),\end{array}$ & HMDB, [22] \\
\hline Quercetin $4^{\prime}$-glucoside & {$[\mathrm{M}-\mathrm{H}]^{-}$} & $\mathrm{C}_{21} \mathrm{H}_{19} \mathrm{O}_{12}$ & 463.0877 & 463.0859 & 3.87 & 5.30 & $\begin{array}{c}343.0383(1.45), 301.0367(100), 283.0280(1.21), 178.9999(6.03) \\
151.0080(2.21), 107.0185(0.65)\end{array}$ & HMDB, METLIN \\
\hline kaempferol galactoside & {$[\mathrm{M}-\mathrm{H}]^{-}$} & $\mathrm{C}_{21} \mathrm{H}_{19} \mathrm{O}_{11}$ & 447.0922 & 447.0940 & 4.03 & 5.36 & $285.0455(100), 284.0392(17.21), 245.088(32.35)$ & HMDB, [22] \\
\hline $\begin{array}{l}\text { Resveratrol-3-o-beta-D- } \\
\text { (sulfonyl)-glucopyranoside }\end{array}$ & {$[\mathrm{M}-\mathrm{H}]^{-}$} & $\mathrm{C}_{20} \mathrm{H}_{21} \mathrm{O}_{11} \mathrm{~S}$ & 469.0799 & 469.0776 & 4.70 & 5.48 & $269.0746(4.56), 227.0669(8.43), 241.0073(100)$ & HMDB, $[2,21]$ \\
\hline Quercetin xyloside & {$[\mathrm{M}-\mathrm{H}]^{-}$} & $\mathrm{C}_{20} \mathrm{H}_{17} \mathrm{O}_{11}$ & 433.0771 & 433.0759 & 2.77 & 5.88 & $\begin{array}{c}301.0384(100), 283.0242(1.57), 271.0315(11.66), 243.0355(1.93), \\
151.0068(5.46)\end{array}$ & HMDB \\
\hline $\begin{array}{l}\text { Hydroxyl } \\
\text { aloe-emodin-o-glucoside }\end{array}$ & {$[\mathrm{M}$} & $\mathrm{O}_{9}$ & 447.0932 & 447.0949 & 3.80 & 6.03 & 241.0542(100), 403.1076(31.52), 197.0674(6.79), 161.0422(1.32) & $\begin{array}{l}\text { [2], HMDB, } \\
\text { METLIN, [21] }\end{array}$ \\
\hline $\begin{array}{l}\text { Resveratrol-3-D-(6 or } \\
\text { 2-galloyl)glucopyranoside }\end{array}$ & {$[\mathrm{M}-\mathrm{H}]^{-}$} & $\mathrm{C}_{27} \mathrm{H}_{25} \mathrm{O}_{12}$ & 541.1341 & 541.1337 & 0.74 & 6.17 & $\begin{array}{c}541.1337(100), 417.093(9.36), 313.0581(25.1), 227.0760(10.23), \\
169.0157(13.53)\end{array}$ & [2], METLIN \\
\hline $\begin{array}{l}\text { Procyanidin-B-1, 3-o-gallate } \\
\text { Kaempferide }\end{array}$ & $\begin{array}{l}{[\mathrm{M}-\mathrm{H}]^{-}} \\
{[\mathrm{M}-\mathrm{H}]^{-}}\end{array}$ & $\begin{array}{l}\mathrm{C}_{37} \mathrm{H}_{29} \mathrm{O}_{16} \\
\mathrm{C}_{16} \mathrm{H}_{11} \mathrm{O}_{6}\end{array}$ & $\begin{array}{l}729.1450 \\
299.0556\end{array}$ & $\begin{array}{l}729.1463 \\
299.0566\end{array}$ & $\begin{array}{l}1.78 \\
3.34\end{array}$ & $\begin{array}{l}6.24 \\
6.43\end{array}$ & $\begin{array}{c}245.0816(19.85), 289.0719(59.57), 407.0833(100), 451.0952(26.42) \\
283.0082(4.81), 271.0251(6.79), 213.0597(100), 151.0082(17.20)\end{array}$ & $\begin{array}{l}\text { [2], HMDB } \\
\text { HMDB }\end{array}$ \\
\hline Apigenin 7-O-glucoside & {$[\mathrm{M}-\mathrm{H}]^{-}$} & $\mathrm{C}_{21} \mathrm{H}_{19} \mathrm{O}_{10}$ & 431.0978 & 431.0995 & 3.94 & 6.69 & 431.1004(100), 311.0561(0.65), 269.0497(92.44), 240.0466(10.54) & $\begin{array}{l}\text { HMDB [2], } \\
\text { METLIN }\end{array}$ \\
\hline Resveratrol & {$[\mathrm{M}-\mathrm{H}]^{-}$} & $\mathrm{C}_{14} \mathrm{H}_{11} \mathrm{O}_{3}$ & 227.0708 & 227.0715 & 3.08 & 6.95 & $\begin{array}{c}\text { 211.0384(6.59), } 185.0640(100), 183.0846(10.63) \text { 159.0824(11.21) } \\
143.0506(80.26), 133.0355\end{array}$ & $\begin{array}{l}\text { Standard, HMDB, } \\
\text { METLIN, [18] }\end{array}$ \\
\hline $\begin{array}{l}\text { Emodin-o-(sulfonyl)- } \\
\text { glucoside }\end{array}$ & {$[\mathrm{M}-\mathrm{H}]^{-}$} & $\mathrm{C}_{21} \mathrm{H}_{19} \mathrm{O}_{13} \mathrm{~S}$ & 511.0541 & 511.0547 & 1.17 & 7.21 & $\begin{array}{c}431.1007(100), 311.0631(1.68), 270.0539(12.13), 269.0485(41.04) \\
257.0494(1.03), 241.0040(4.82), 225.0545(0.82)\end{array}$ & [2], METLIN, [21] \\
\hline Torachrysone 8-glucoside & {$[\mathrm{M}-\mathrm{H}]^{-}$} & $\mathrm{C}_{20} \mathrm{H}_{23} \mathrm{O}_{9}$ & 407.1342 & 407.1353 & 2.70 & 7.86 & $245.0811(100), 269.0744(0.4)$ & $\begin{array}{l}\text { METLIN, } \\
\text { HMD, [21] }\end{array}$ \\
\hline Luteolin & {$[\mathrm{M}-\mathrm{H}]^{-}$} & $\mathrm{C}_{15} \mathrm{H}_{9} \mathrm{O}_{6}$ & 285.0394 & 285.0387 & 2.46 & 8.04 & $\begin{array}{c}\text { 285.0381(100), 267.0342(2.78), 257.0439(1.03),243.0279(1.84), } \\
241.0489(2.14), 229.0493,217.0488(3.9), 175.0381(5.29), \\
151.0031(11.51), 133.0329(18.27), 121.0273(0.73), 107.0162(3.31)\end{array}$ & $\begin{array}{c}\text { HMDB, } \\
\text { METLIN, [2,24] }\end{array}$ \\
\hline Emodin-6-o-glucoside & {$[\mathrm{M}-\mathrm{H}]^{-}$} & $\mathrm{C}_{21} \mathrm{H}_{19} \mathrm{O}_{10}$ & 431.0978 & 431.0982 & 0.93 & 8.14 & $\begin{array}{c}431.1000(48.67), 311.0584(4.54), 293.0518(1.66), 282.0597(0.7) \\
269.0497(100), 225.0582(2.41)\end{array}$ & HMDB, [21] \\
\hline $\begin{array}{l}\text { Torachrysone-8-o-(sulfonyl)- } \\
\text { glucoside }\end{array}$ & {$[\mathrm{M}-\mathrm{H}]^{-}$} & $\mathrm{C}_{20} \mathrm{H}_{23} \mathrm{O}_{12} \mathrm{~S}$ & 487.0905 & 487.0892 & 2.67 & 8.41 & $\begin{array}{c}\text { 407.1355, 287.0927(1.03), 245.0841(100), 243.0051(8.32), } \\
\text { 241.0076(90.07), 230.0601(7.32), 215.0435(0.55), 113.0251(1.32) }\end{array}$ & {$[2,21,22]$} \\
\hline Hydroxyl aloe-emodin & {$[\mathrm{M}-\mathrm{H}]^{-}$} & $\mathrm{C}_{15} \mathrm{H}_{9} \mathrm{O}_{6}$ & 285.0394 & 285.0387 & 2.46 & 8.93 & $\begin{array}{l}\text { 285.0401(100), 241.0543(2.17), 211.0421(0.34), 197.0586(0.69), } \\
\text { 195.0533(0.4), 167.05(0.33), 151.0073(10.22), 133.0319(14.97), }\end{array}$ & HMDB, [21] \\
\hline
\end{tabular}


Table 1. Cont.

\begin{tabular}{|c|c|c|c|c|c|c|c|c|}
\hline Identification & $\begin{array}{l}\text { Precursor } \\
\text { Ion }\end{array}$ & $\begin{array}{l}\text { Molecular } \\
\text { Formula }\end{array}$ & $\begin{array}{l}\text { Predicted } \\
\quad m / z\end{array}$ & $\begin{array}{l}\text { Measured } \\
\quad m / z\end{array}$ & PPM & RT & ms2 Fragment Ions at Negative Mode & References \\
\hline 6"-Malonylgenistin & {$[\mathrm{M}-\mathrm{H}]^{-}$} & $\mathrm{C}_{24} \mathrm{H}_{21} \mathrm{O}_{13}$ & 517.0982 & 517.0983 & 019 & 9.05 & $\begin{array}{c}\text { 473.1056(100), 455.0952(0.53), 431.0968(2.51), 311.0553(3.47), } \\
\text { 269.0454(75.44), 413.0835(0.25) }\end{array}$ & [22], HMDB \\
\hline Apigenin & {$[\mathrm{M}-\mathrm{H}]^{-}$} & $\mathrm{C}_{15} \mathrm{H}_{9} \mathrm{O}_{5}$ & 269.0450 & 269.0449 & 0.37 & 9.10 & $\begin{array}{c}\text { 269.0494(100), 241.0493(2.97), 227.0409(3.04), 225.0536(36.73), } \\
151.0063(1.39),\end{array}$ & $\begin{array}{l}\text { METLIN, } \\
\text { HMDB, [25] }\end{array}$ \\
\hline $\begin{array}{c}\text { Apigenin } \\
\text { 7-O-(6"-O-acetylglucoside) }\end{array}$ & {$[\mathrm{M}-\mathrm{H}]^{-}$} & $\mathrm{C}_{23} \mathrm{H}_{21} \mathrm{O}_{11}$ & 473.1084 & 473.1086 & 0.42 & 9.15 & $\begin{array}{c}\text { 473.1107(100), 431.0898(10.92), } 413.0669(10.97), 311.0626(16.45), \\
269.0460(37.55),\end{array}$ & HMDB \\
\hline Bergapten & {$[\mathrm{M}-\mathrm{H}]^{-}$} & $\mathrm{C}_{12} \mathrm{H}_{7} \mathrm{O}_{4}$ & 215.0344 & 215.0351 & 3.26 & 9.23 & $171.0469(2.83), 159.0484(100), 143.0535(18.1), 131.0531(86.87)$ & HMDB, METLIN \\
\hline Torachrysone-8-o-(acetyl)-glucos & $\mathrm{idA}-\mathrm{H}]^{-}$ & $\mathrm{C}_{22} \mathrm{H}_{25} \mathrm{O}_{10}$ & 449.1442 & 449.1439 & 0.45 & 9.24 & $269.0438(2.47), 245.0821(100), 230.0583(11.11), 215.0363(2.21)$, & [2], HMDB \\
\hline Acetylemodin-o-glucoside & {$[\mathrm{M}-\mathrm{H}]^{-}$} & $\mathrm{C}_{23} \mathrm{H}_{21} \mathrm{O}_{11}$ & 473.1078 & 473.1073 & 1.06 & 9.54 & $\begin{array}{c}335.9747(3.94), 311.0626(16.45), 283.0506(5.9), 269.0460(100), \\
267.07(12.37)\end{array}$ & {$[2,21]$} \\
\hline $\begin{array}{l}\text { Physcion-8-glucoside } \\
6^{\prime \prime} \text {-O-Acetylglycitin }\end{array}$ & $\begin{array}{l}{[\mathrm{M}-\mathrm{H}]^{-}} \\
{[\mathrm{M}-\mathrm{H}]^{-}}\end{array}$ & $\begin{array}{l}\mathrm{C}_{22} \mathrm{H}_{21} \mathrm{O}_{10} \\
\mathrm{C}_{24} \mathrm{H}_{23} \mathrm{O}_{11}\end{array}$ & $\begin{array}{l}445.1129 \\
487.1240\end{array}$ & $\begin{array}{l}445.1145 \\
487.1240\end{array}$ & $\begin{array}{c}3.59 \\
0\end{array}$ & $\begin{array}{c}9.71 \\
11.35\end{array}$ & $\begin{array}{c}\begin{array}{c}325.0434(2.31), \\
283.0630(100), \\
283.0645(100), 267.0445(14.86), \\
267.0868(1.18)\end{array}\end{array}$ & $\begin{array}{l}{[18,21]} \\
\text { HMDB }\end{array}$ \\
\hline 6-hydroxylrhein & {$[\mathrm{M}-\mathrm{H}]^{-}$} & $\mathrm{C}_{15} \mathrm{H}_{7} \mathrm{O}_{7}$ & 299.0186 & 299.0187 & 0.33 & 11.48 & $\begin{array}{c}300.0192(32.33), 299.0193(100), 255.0328(16.88), 227.0381(6.13), \\
211.0423(12.13), 199.0473(1.99)\end{array}$ & {$[2,21]$} \\
\hline Formononetin 7-O-glucoside & {$[\mathrm{M}-\mathrm{H}]^{-}$} & $\mathrm{C}_{22} \mathrm{H}_{21} \mathrm{O}_{9}$ & 429.1186 & 429.1175 & 2.56 & 12.18 & $\begin{array}{c}\text { 411.0650(7.35), 307.0586(7.87), 293.0452(100), 267.0667(21.06), } \\
237.0614(6.34)\end{array}$ & HMDB, METLIN \\
\hline Acetylemodin & {$[\mathrm{M}-\mathrm{H}]^{-}$} & $\mathrm{C}_{17} \mathrm{H}_{11} \mathrm{O}_{6}$ & 311.0551 & 311.0560 & 3.11 & 12.79 & $\begin{array}{c}311.0563(100), 269.0429(14.3), 268.0402(48.88), 240.0466(3.84), \\
224.0481(1.22), 196.0565(1.02)\end{array}$ & {$[2,21]$} \\
\hline Apigenin 7-sulfate & {$[\mathrm{M}-\mathrm{H}]^{-}$} & $\mathrm{C}_{15} \mathrm{H}_{9} \mathrm{O}_{8} \mathrm{~S}$ & 349.0018 & 349.0034 & 4.58 & 12.81 & $\begin{array}{c}331.2035(0.19), 306.3984(0.17), 269.0437(100), 241.0124(0.71), \\
227.0115(0.45)\end{array}$ & HMDB, METLIN \\
\hline Emodin & {$[\mathrm{M}-\mathrm{H}]^{-}$} & $\mathrm{C}_{15} \mathrm{H}_{9} \mathrm{O}_{5}$ & 269.0450 & 269.0460 & 3.71 & 15.00 & $\begin{array}{c}\text { 241.0512(21.33), 225.0546(100), } 197.0626(1.11), 181.0666(1.33), \\
157.0626(0.39), 65.0044(0.14)\end{array}$ & $\begin{array}{l}\text { Standard, [18], } \\
\text { HMDB, [21] }\end{array}$ \\
\hline Physcion & {$[\mathrm{M}-\mathrm{H}]^{-}$} & $\mathrm{C}_{16} \mathrm{H}_{11} \mathrm{O}_{5}$ & 283.0606 & 283.0611 & 1.77 & 15.39 & $\begin{array}{c}269.0381(24.64), 268.0362(100), 241.0456(8.91), 240.0412(21.34), \\
205.0512(13.4), 197.2618(1.65)\end{array}$ & METLIN, [21] \\
\hline
\end{tabular}




\subsubsection{Stilbenes and Their Derivatives}

Stilbenes are the major active components in P. cuspidatum and the quality of P. cuspidatum is usually evaluated based on their contents. In this study, stilbenes and their derivatives were tentatively identified from different tissues of P. cuspidatum. Stilbenes and their aglycones share the same fragment ion at $227 \mathrm{~m} / z$, the $[\mathrm{M}-\mathrm{H}]^{-}$ion of 3,5,4'-trihydroxy-stilbene. For example, the molecular ion M227T7 was eluted at $6.95 \mathrm{~min}$ with an $\mathrm{m} / \mathrm{z}$ value of $227.0720\left([\mathrm{M}-\mathrm{H}]^{-}\right)$, which indicated it was the molecular ion of $\mathrm{C}_{14} \mathrm{H}_{11} \mathrm{O}$. The fragment ions of M227T7 occurred at 185.0624, 159.0824, 143.0506 and 107.0522 $\mathrm{m} / \mathrm{z}$ in negative ion mode. Fragments 185.0624 and 143.0506 were formed by successive losses of $\mathrm{C}_{2} \mathrm{H}_{2} \mathrm{O}$ groups from 227.07 [18]. These fragments and retention times were fitted to the resveratrol standard. Therefore, compound M227T7 was identified as resveratrol. The molecular ions of M389T5 had $m / z$ values of $389.1236\left([\mathrm{M}-\mathrm{H}]^{-}\right)$and $435.1279\left(\left[\mathrm{M}+\mathrm{HCOO}^{-}\right.\right.$, which corresponded to the molecular formulae of $\mathrm{C}_{20} \mathrm{H}_{21} \mathrm{O}_{8}$ and $\mathrm{C}_{21} \mathrm{H}_{23} \mathrm{O}_{10}$, respectively. The ion fragments of M389T5 included a fragment with an $m / z$ value of 227.0930, which was the same as resveratrol. Thus, a glucoside was cleaved and the precursor ion M389T5 lost a glucose unit. Most resveratrol fragments were detected in the MS/MS analysis of M389T5. The retention time of M389T5 was $5.09 \mathrm{~min}$, which was the same as the piceid reference standard. Therefore, this compound was identified as piceid. The proposed fragmentation pathways for piceid and resveratrol are illustrated in Figure 2.

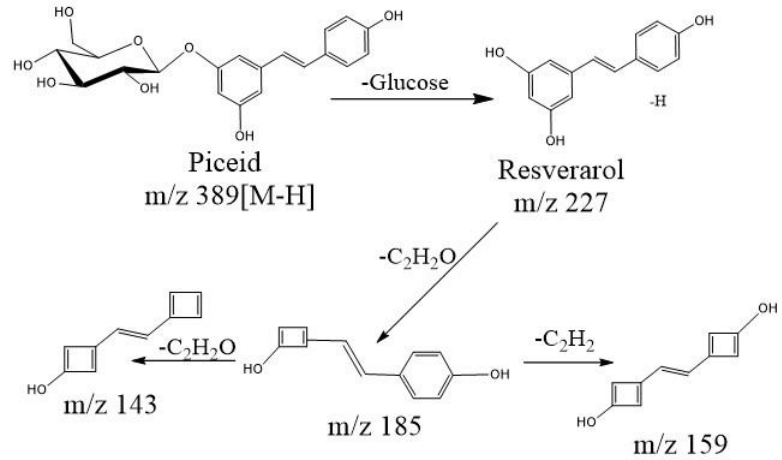

(a)

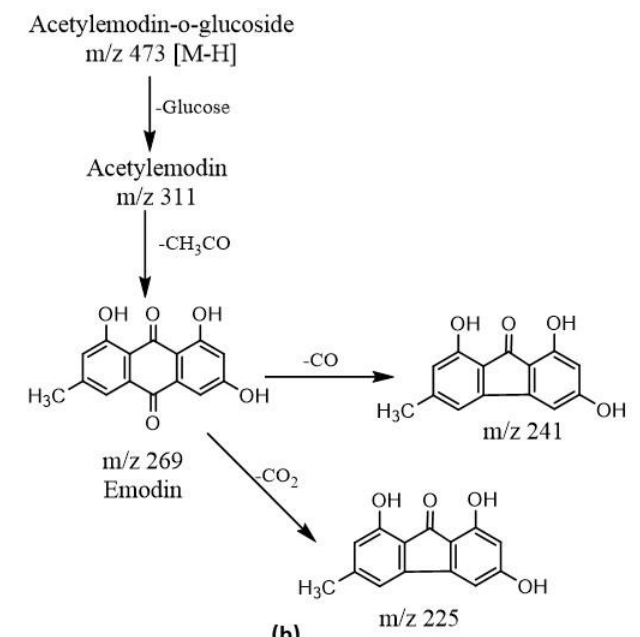

(b)

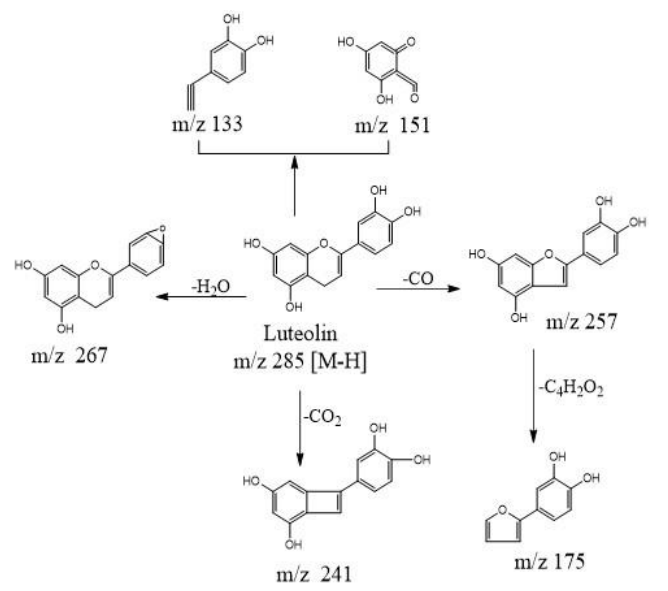

(c)

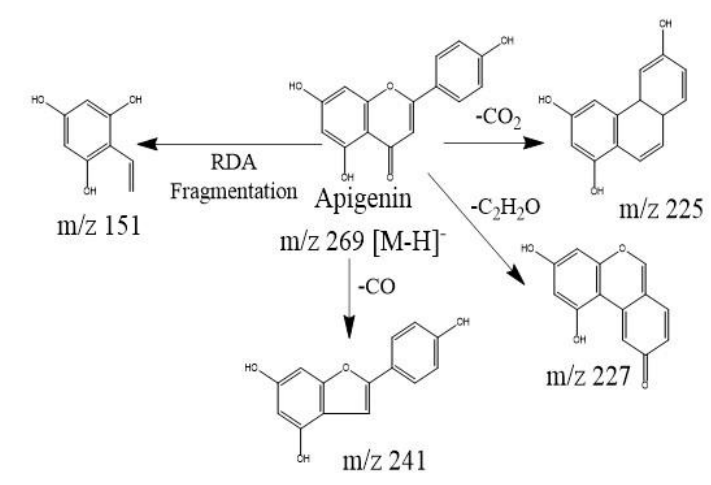

(d)

Figure 2. The proposed fragmentation pathways of selected compounds. (a) Piceid and resveratrol, (b) Acetylemodin-o-glucoside and emodin, (c) Luteolin, (d) Apigenin. The proposed fragmentation pathways were established based on generated fragment ions. 
The other stilbene compounds displayed the deprotonated $[\mathrm{M}-\mathrm{H}]^{-}$and shared the same $m / z$ 227.0767 fragment. These compounds were tentatively identified by comparing the fragments with the references and databases, including METLIN and HMDB. For example, the ion M541T6 ( $m / z$ 541.1379, retention time $6.20 \mathrm{~min}$ ) was tentatively identified as resveratrol-3-D-(6-galloyl) glucopyranoside on the basis of the reference [2]. The stilbene contents were significantly greater in root and rhizome than in other tissues (Figure 3), which was consistent with previous studies. In addition, these results support the use of P. cuspidatum root and rhizome in 'Traditional Chinese Medicines'.

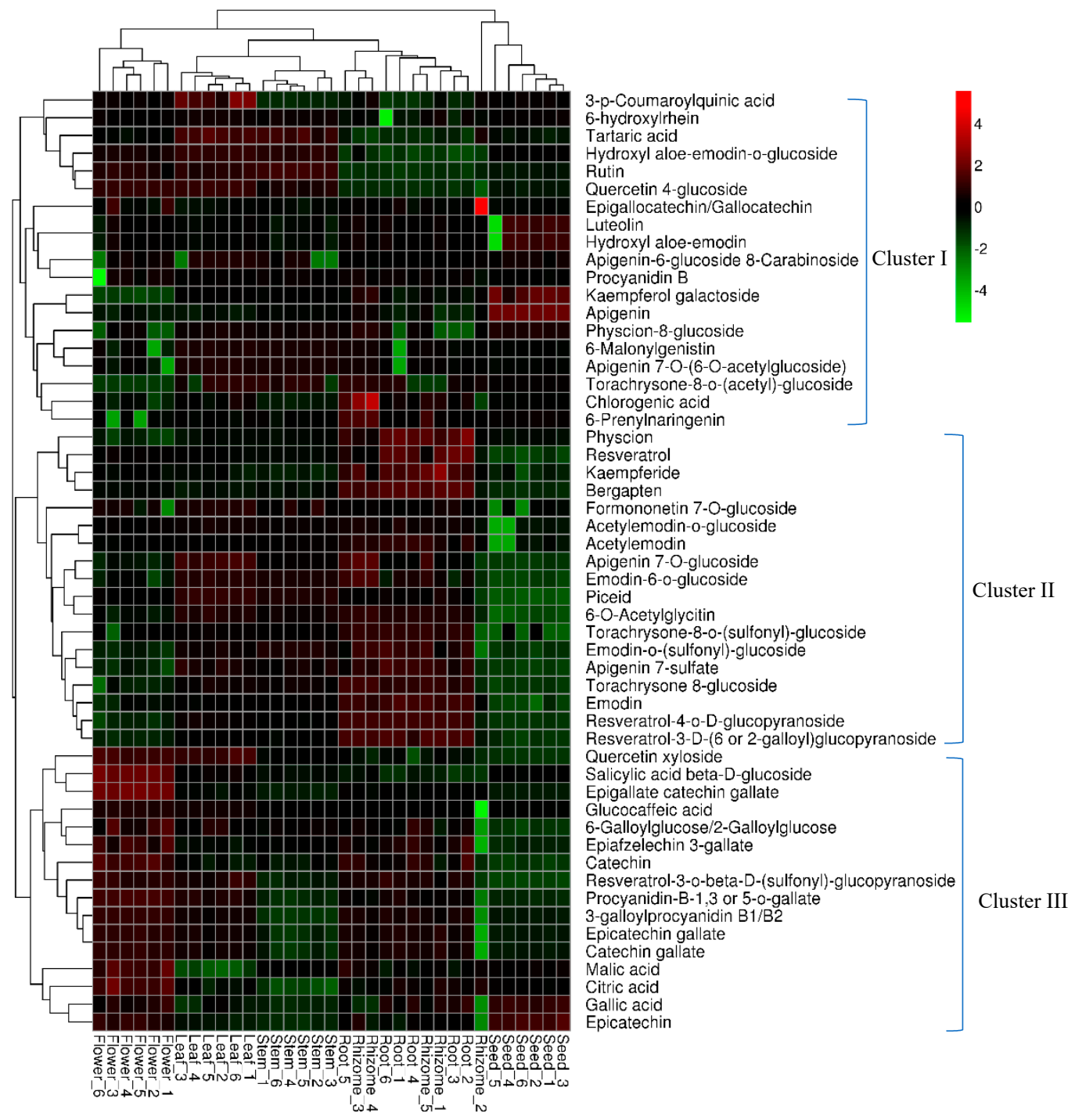

Figure 3. Heatmap analysis of the relative distributions of 53 identified metabolites in different tissues. The heatmap was exhibited using a hierarchical clustering algorithm based on the normalized average signal abundance. The red and green color in the heatmap represent an increase and a decrease of metabolite level, respectively.

\subsubsection{Anthraquinones}

P. cuspidatum is a rich source of anthraquinone and its derivatives, which have many biological activities, such as anti-cancer, anti-inflammatory and anti-methicillin-resistant Staphylococcus aureus [26]. In this study, nine anthraquinone compounds, including emodin and its derivatives, were tentatively identified according to the accurate $m / z$ values (within a mass error of $5 \mathrm{ppm}$ ) and characteristic fragments. 1,8-Dihydroxy-9,10-anthraquinone is the common core of anthraquinones and its deprotonation can generate a characteristic fragment ion with an $m / z$ value of 269.0450 . The negative 
ion MS/MS fragments of M269T15 occurred at 241.0512, 225.0568, 197.0621, 181.0666, 115.0552 and $65.0044 \mathrm{~m} / \mathrm{z}$. The fragments at 241.0521 and $225.0568 \mathrm{~m} / \mathrm{z}$ of M269T15 were generated through the loss of $\mathrm{CO}$ and $\mathrm{CO}_{2}$, respectively. Aloe emodin and emodin are a pair of isomers and have different ion MS/MS fragment distributions. Emodin has a characteristic fragment of $225.05 \mathrm{~m} / \mathrm{z}$, while that of aloe emodin occurs at $239.03 \mathrm{~m} / z$ [18,21]. The retention time of M269T15 was $15.55 \mathrm{~min}$ and was consistent with the emodin reference standard. Therefore, M269T15 was identified as emodin. The proposed fragmentation pathways for emodin are shown in Figure 2.

The ion $m / z$ values of physcion, physcion-8-glucoside and emodin-6-O-glucoside were tentatively identified by matching MS/MS spectra with those reported in the literature and databases. M283T15 was identified as physcion, and the precursor molecule's $m / z$ value was 283.0611 in the negative ion mode. Its fragments occurred at $268.0362,240.0412$ and $205.0501 \mathrm{~m} / \mathrm{z}$, which are characteristic of physcion. These fragments at 268.0362 and $240.0412 \mathrm{~m} / \mathrm{z}$ were generated by the successive losses of a $\mathrm{CH}_{3}$ and a $\mathrm{CO}$ group $[18,21]$. The glucosides of physcion were also identified using the above method. For example, M445T10 was identified as physcion-8-glucoside. The MS/MS fragments occurred at 445.1145, 283.0630, 240.0445 and $165.0464 \mathrm{~m} / z$. Fragment $283.0630\left([\mathrm{M}-\mathrm{H}]^{-}\right) \mathrm{m} / z$ represented the ion of physcion, and the fragment at $240.0445 \mathrm{~m} / \mathrm{z}$ was characteristic of physcion [27].

\subsubsection{Flavonoids, Isoflavonoids and Other Compounds}

Flavonoids are a large family of compounds with a variety of bioactive effects, such as antioxidant and anti-inflammatory activities, and play important roles in the coloration of flowers and ripening fruit [28]. In this study, 25 compounds were putatively identified as flavonoids and flavonoid glucosides by comparing the accurate $m / z$ values and typical fragments with databases, literature and standards.

Compound ion M289T3 was identified as catechin with a precursor molecular $[\mathrm{M}-\mathrm{H}]^{-}$ion at $289.0737 \mathrm{~m} / \mathrm{z}$. The MS/MS fragments occurred at 271.0656, 245.0885, 221.0838 and $151.04 \mathrm{~m} / \mathrm{z}$. The retention time of M289T3 (2.64 min) and the fragment mode were the same as those of the reference standard. Epicatechin-gallate and rutin were identified by analyzing the fragments against metabolomics databases and references [23]. Molecular ion M269T9 was tentatively identified as apigenin. The $m / z$ value of M269T9 was 269.0449, with a retention time of $9.10 \mathrm{~min}$. Fragments of M269T9 occurred at 269.0494, 227.0409, 225.0536 and $151.0063 \mathrm{~m} / \mathrm{z}$. Ions of 227 ([M $-\mathrm{H}-$ $\left.\left.\mathrm{C}_{2} \mathrm{H}_{2} \mathrm{O}\right]^{-}\right)$and $225\left(\left[\mathrm{M}-\mathrm{H}-\mathrm{CO}_{2}\right]^{-}\right)$were generated by losing a $\mathrm{C}_{2} \mathrm{H}_{2} \mathrm{O}$ group and a $\mathrm{CO}_{2}$ group, respectively. The ion fragment at $151.00 \mathrm{~m} / \mathrm{z}$ was formed by the cleavage of the $\mathrm{C}$-ring [29]. There was no vicinal-hydroxyl group in apigenin, but it was not the result of the neutral loss of a $\mathrm{H}_{2} \mathrm{O}$ group. Thus, an ion at $251 \mathrm{~m} / z\left(\left[\mathrm{M}-\mathrm{H}-\mathrm{H}_{2} \mathrm{O}\right]^{-}\right)$was not found in the MS/MS spectrum of apigenin [30]. The MS/MS spectrum of M269T9 was in accordance with the fragments of apigenin [25]; therefore, it was tentatively identified as apigenin. Similarly, M349T13 and M431T7 were tentatively identified as apigenin 7-sulfate and apigenin 4-O-glucoside, respectively.

Luteolin was also identified by analyzing the fragments of M285T8. The ion fragments $\mathrm{m} / \mathrm{z}$ occurred at 285.0381, 267.0342, 257.0439, 243.0279, 241.0489, 229.0493, 217.0488, 175.0381, 151.0031, $133.0329,121.0273$ and $107.0162 \mathrm{~m} / z$. Luteolin has a vicinal-hydroxyl group, and it can generate ion ( $\left[\mathrm{M}-\mathrm{H}-\mathrm{H}_{2} \mathrm{O}\right]^{-}$) at $267 \mathrm{~m} / z$ by losing a $\mathrm{H}_{2} \mathrm{O}$ group. The ions at 257 and $241 \mathrm{~m} / z$ were formed by losing a $\mathrm{CO}$ and $\mathrm{CO}_{2}$ group, respectively. The two fragments at 151.00 and $133.03 \mathrm{~m} / z$ were generated through retro-Diels-Alder cleavage, which is the characteristic flavonoids pathway [24,31]. With this information on the fragment ions, M285T8 was tentatively identified as luteolin [29,30]. The proposed fragmentation pathways for apigenin and luteolin are illustrated in Figure 2. Other compounds included tannins, such as gallic acid and bergapten. These compounds were also tentatively identified using databases and references. We also identified the compounds luteolin, epigallate catechin gallate, apigenin-6-glucoside, kaempferide, formononetin 7-O-glucoside, bergapten, $6^{\prime}$-malonylgenistin and procyanidin-B-1, 3-O-gallate for the first time, to our knowledge, in this species. 


\subsection{Global Distribution and Relative Quantitative Analysis of Identified Metabolites}

The distribution and relative quantification of the identified metabolites from different tissues of P. cuspidatum were illustrated using a heatmap (Figure 3). The heatmap was constructed using a hierarchical clustering algorithm based on area-normalized metabolite quantities. The red and green colors in the heatmap represent higher and lower relative contents than the average value, respectively. In the heatmap, root and rhizome samples formed a group, except for sample "rhizome 2", which was assigned to the seed group. Other samples from different tissues were clearly separated, suggesting that the metabolites were significantly different among tissues. As described in Figure 3, the relative abundance levels of the 53 compounds from different tissues were divided into three clusters. Cluster I mainly contained flavonoids, including apigenin, luteolin, rutin and apeginin-7-O-glucoside. The greater relative contents of these compounds were found in the leaf and stem groups. However, the other compounds, including 6-prenylnaringenin, gingerol, chlorogenic acid, and torachrysone 8-glucoside, were present at greater levels in rhizomes. Apigenin and luteolin are present in a variety of fruit, vegetables and medicinal plants, and play multiple roles in development and plant-environment interactions. Apigenin and its glycosides are present in seeds of Lupinus, and apigenin can induce the antioxidant defense system in seedlings [32,33]. In this study, apigenin and luteolin were detected in P. cuspidatum and their relative contents in seeds were greater than in other tissues. Apigenin might enhance antioxidant activities in seeds and improve the germination rate of P. cuspidatum. Apigenin and luteolin have many beneficial effects on human health, including antitumor, anti-inflammatory, neuroprotective effects, and biological organ protection and immune regulation [34,35]. Thus, these results provide insights into the possible exploitation of P. cuspidatum seeds to improve human health. The rutin and quercetin 4-glucoside contents were greater in leaves, stems and flowers than in other tissues. This might explain why the leaves and stems of P. cuspidatum are used as medicine and food.

Cluster II mainly contained anthraquinones, stilbenes and other compounds, such as torachrysone 8-glucoside, bergapten and kaempferide. Stilbenes and anthraquinones, including resveratrol, emodin and their derivatives, had much greater abundance levels in roots and rhizomes than in other tissues. This could be the basis of why the root and rhizome of P. cuspidatum are categorized as medicinal tissues in Chinese Pharmacopoeia. The concentrations of these compounds in the seeds and flowers were lower than in other tissues. Torachrysone 8-glucoside and torachrysone-8-O-(acetyl)-glucoside were either not detectable or present at extremely low levels in flowers and seeds.

Catechin, epicatechin, quercetin xyloside, epigallate catechin gallate, epiafzelechin 3-gallate, 3-galloylprocyanidin B1 or B2 and procyanidin-B-1, 3-O-gallate were mainly present in Cluster III. These compounds were present in greater concentrations in flowers than in other tissues. The accumulation of these compounds in flowers could protect the plant from pathogen attacks and abiotic stresses [36,37]. Karakaya reported a similar result, namely that the flowers of Z. absinthifolia can be a potential resource of natural antioxidant compounds [38]. The abundance levels of the other compounds in this cluster, except gallic acid and quercetin xyloside, were lower in seeds than in other tissues. These compounds have many benefits to human health. For example, epigallate catechin gallate could be used to treat cyclic GMP-AMP synthase mediated autoimmune diseases [39]. Thus, the flowers of P. cuspidatum could be further processed into tea or herbal beverages.

\subsection{Identification of Chemical Markers}

Metabolomics and multivariate statistical analyses, including orthogonal partial least square discriminant analysis (OPLS-DA), have become powerful tools to distinguish the differences among metabolite patterns and reveal marker compounds in different samples [40,41]. Mo et al. used UPLC-MS-based untargeted metabolomics profiling to discriminate samples from different parts of Panax notoginseng [42]. Li et al. established an UPLC-QTOF-MS-based metabolomics approach and found eight markers that played significant roles in the bioactivities of branch extracts of Garcinia oblongifolia [43]. The roots and rhizomes are the main medicinal parts of P. cuspidatum according to Chinese Pharmacopoeia, but the chemical differences among different tissues remains unknown. 
OPLS-DA was used to investigate the differential compounds among tissue groups. The score and loading plots of OPLA-DA are presented in Figure 4. The score plots and explained variances of the OPLS-DA model were similar to those of the PCA plots. The $\mathrm{R}^{2} \mathrm{X}, \mathrm{R}^{2} \mathrm{Y}$ and $\mathrm{Q}^{2}$ values were 0.726 , 0.987 and 0.973 , respectively. These parameters suggested that the model was a good fit and had a satisfactory predictive capability.

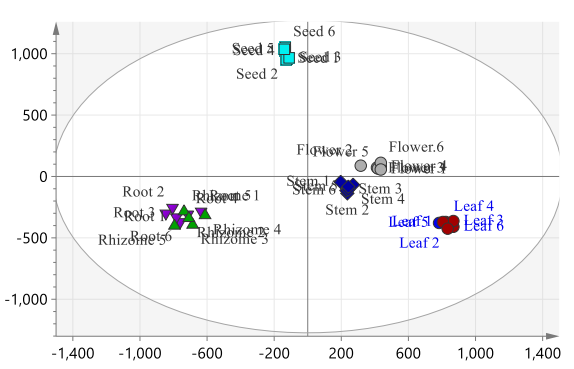

(a)

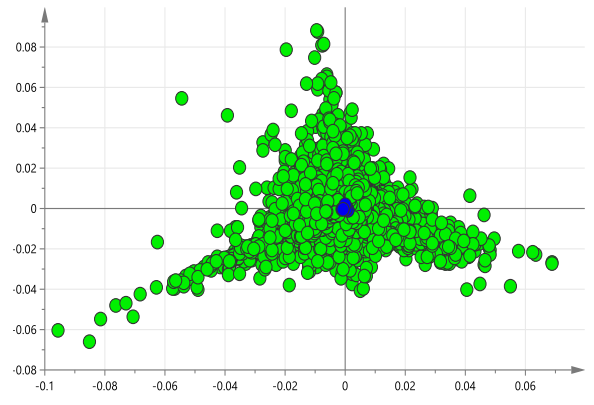

(b)

Figure 4. OPLS-DA score plots and loading plots. (a) Score plots; (b) loading plots. The $\mathrm{R}^{2} \mathrm{X}, \mathrm{R}^{2} \mathrm{Y}$ and $\mathrm{Q}^{2}$ values of OPLS-DA were $0.726,0.987$ and 0.973 , respectively.

The potential chemical markers that could distinguish different tissues of $P$. cuspidatum were selected using OPLS-DA and putatively identified. The marker ions were screened according to the OPLS-DA loading plots, the VIP value $(>1)$ and the $P$ value $(<0.001)$, and the following 13 potential chemical marker ions were tentatively identified: emodin, quercetin $4^{\prime}$-glucoside, epiafzelechin 3-gallate, epicatechin, citric acid, luteolin, apigenin, apigenin 7-O-glucoside, hydroxyl aloe-emodin-o-glucoside, catechin gallate, torachrysone 8-glucoside, physcion and resveratrol. The relative abundances of these compounds are displayed in Figure 5. The contents of most of the compounds differed significantly among different groups; consequently, they might be used as markers for differentiating tissues of P. cuspidatum. The relative contents of emodin, torachrysone 8-glucoside, resveratrol and physcion were as follows: roots/rhizomes $>$ leaves $>$ stems $>$ flowers $>$ seeds. Thus, these compounds could be used to distinguish the root/rhizome from other tissues of P. cuspidatum. The greatest contents of luteolin, apigenin and epicatechin were found in seeds and could be used as markers to distinguish seed from other tissues. The citric acid, epiafzelechin 3-gallate and catechin gallate contents were greater in flowers than in other tissues, while those of the hydroxyl aloe-emodin-O-glucoside, quercetin $4^{\prime}$-glucoside and catechin gallate were greater in leaves than in other tissues.

The identification of these potential markers provided useful information for the further isolation of functional genes in combination with transcriptome or genome data, and they could be applied to discriminate among the different tissues of $P$. cuspidatum. Our results provide some guidance for the further exploitation of P. cuspidatum, in particular the different tissues of this medicinal herb. By combining these results with transcriptome data, it may be possible to identify functional genes correlated with the biosynthesis of pharmaceutical compounds. 


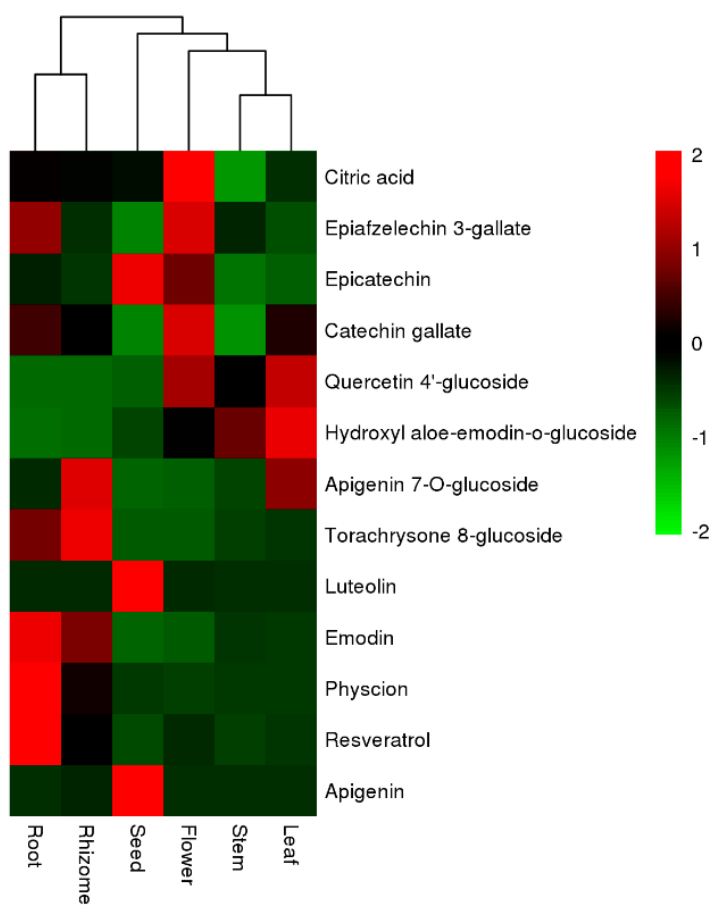

Figure 5. The relative contents of thirteen markers in different tissues of P. cuspidatum. The contents of each compound was normalized as a relative content. The heatmap was exhibited using a hierarchical clustering algorithm based on the normalized average abundance.

\section{Materials and Methods}

\subsection{Plant Materials and Chemicals}

The root, rhizome, stem, leaf, flower and seed of P. cuspidatum grown in the Institute of Botany (Beijing, China), the Chinese Academy of Sciences, were harvested for metabolite extraction. Tissues for each biological replicate sample were collected from three individual plants. All samples were frozen immediately and then transferred to $-80^{\circ} \mathrm{C}$ for storage until analysis.

All chemical standards used in this study were purchased from Sigma-Aldrich (St. Louis, MO. USA). HPLC grade methanol, acetonitrile and formic acid were obtained from Merck (Darmstadt, Germany). Ultrapure water was generated by a Milli-Q system (Millipore, MA, USA).

\subsection{Metabolite Extractions}

Samples with biological replicates were prepared for each tissue group. Tissue samples were ground to a powder using a pre-chilled mortar and pestle, and then freeze-dried. Briefly, $50 \mathrm{mg}$ freeze-dried plant tissue was weighed and transferred into a 2-mL centrifuge tube. Then, $1 \mathrm{~mL}$ $\mathrm{MeOH}-\mathrm{H}_{2} \mathrm{O}(80: 20, v / v)$ was added, vortexed vigorously three times, and an ultrasonic-assisted extraction was performed for $30 \mathrm{~min}$ in an ice bath. Samples were extracted overnight at $4{ }^{\circ} \mathrm{C}$ and then centrifuged for $15 \mathrm{~min}$ at $12,000 \times \mathrm{g}$. Afterward, $500 \mu \mathrm{L}$ clear supernatant per extract was filtered through a $0.22-\mu \mathrm{m}$ PTFE filter before the LC-MS analysis.

\subsection{LC-MS Analysis}

The metabolomics analysis was performed using an Agilent 1290 HPLC system coupled with a Q-TOF ESI mass spectrometer (Agilent, Santa Clara, CA, USA). HPLC separation was performed using a Phenomenex reversed-phase C18 column (Kinetex, $2.6 \mu \mathrm{m}, 100 \mathrm{~mm} \times 2.1 \mathrm{~mm}$, Phenomenex Inc., Torrance, CA, USA). Water supplemented with $0.1 \%$ formic acid (A) and acetonitrile supplemented with $0.1 \%$ formic acid (B) were used as mobile phases at a flow rate of $0.4 \mathrm{~mL} / \mathrm{min}$. The gradient was optimized as follows: $0-1 \mathrm{~min}$ : $5 \% \mathrm{~B}$; $1-6 \mathrm{~min}$ : 5\%-23\% B; $6-10 \mathrm{~min}$ : $23 \%-29 \% \mathrm{~B} ; 10-18 \mathrm{~min}$ : 
29\%-70\% B; 18-26 min: 70\%-98\% B; 26-29 min: 98\% B; 29-30 min: 98\%-5\% B; and 30-33 min: 5\% B. In all cases, the injected volume was $10 \mu \mathrm{L}$, and the column temperature was maintained at $30{ }^{\circ} \mathrm{C}$. An Agilent 6530 high-resolution Q-TOF mass spectrometer (Agilent, Santa Clara, CA, USA) was used to collect MS data in negative ion mode. The parameters were as follows: mass range: $50-1000 \mathrm{~m} / \mathrm{z}$; dry gas temperature: $300{ }^{\circ} \mathrm{C}$; dry gas flow rate: $5 \mathrm{~L} / \mathrm{min}$; nebulizer: 35 psi; capillary voltage: $3500 \mathrm{~V}$; fragmentor: $135 \mathrm{~V}$; skimmer: $65 \mathrm{~V}$; full MS scan at a resolution of 20,000 was used. Two scans were obtained per s to provide data points for relative quantification. An MS2 scan was carried out using the data-dependent mode with a collision energy of $20 \mathrm{~V}$.

\subsection{Metabolomics Profiles and MS Data Analysis}

High-resolution full-scan MS and MS/MS data were analyzed using MassHunter qualitative analysis software (Agilent) (Agilent Technologies Inc., Palo Alto, CA, USA) and XCMS online (https: //xcmsonline.scripps.edu/, Accessed 14.12.18). The original data files (.d) were converted to mzXML format using ProteoWizard (ProteoWizard, Palo Alto, CA, USA). Then, the mzXML files' data of each group were loaded and analyzed by XCMS using a standard XCMS workflow. Parameter settings for XCMS processing were as follows: minimum peak width $=10 \mathrm{~s}$, maximum peak width $=60 \mathrm{~s}$, mzwid $=0.015, \operatorname{minfrac}=0.5, \mathrm{bw}=5$ and signal $/$ noise threshold $=6$. Then, the peak detection, retention time correction, chromatogram alignment and statistical evaluation were performed. The results contained a peak list with $m / z$ values, peak intensity fold changes, statistical significances ( $p$ value), retention times and extracted peak intensities. These results were used for the subsequent multivariate statistical analysis.

A principal component analysis (PCA) and orthogonal partial least square discriminant analysis (OPLS-DA) were carried out using SIMCA-P 13 software (Umetrics, Umea, Sweden) for unsupervised and supervised multivariate analyses, respectively. Variables of the data sets were pareto-scaled and mean-centered before the multivariate analysis. Parameters of the PCA and OPLS-DA, including the goodness-of-fit parameter $R^{2} X$, the variance of the response variable $R^{2} Y$ and the predictive ability parameter $\mathrm{Q}^{2}$, were also calculated for model evaluation. Furthermore, a hierarchical cluster heatmap was constructed using Biomarker Cloud (http:/ / www.biocloud.net/. Accessed 14.12.18).

Metabolites were identified and characterized based on high-resolution MS-associated methods, which included comparing the results with databases and the literature, and comparing the retention times and fragments with those of standards. Metabolites were identified using the accurate high-resolution $m / z$ values and MS/MS fragment patterns. First, the accurate $m / z$ values were searched against compound databases KNApSAcK (http:/ / kanaya.naist.jp/KNApSAcK/. Accessed 14.12.18), BATMAN-TCM (http://bionet.ncpsb.org/batman-tcm/. Accessed 14.12.18), METLIN (https://metlin.scripps.edu/. Accessed 14.20.18) and HMDB (http://www.hmdb.ca/. Accessed 14.12.18), with a mass accuracy of $10 \mathrm{ppm}$, a list of chemical formulae was generated. Then, the MS/MS spectra were analyzed by comparing both fragment patterns and isotope ratios to identify the metabolites [44].

\section{Conclusions}

In this study, the tissue-specific metabolite variations in Polygonum cuspidatum were investigated using an LC-MS-based metabolomics approach for the first time. A total of 53 bioactive compounds were identified, 8 for the first time in this species. In addition, the relative abundance levels of these compounds in different tissues were also measured. The phytochemical composition variations of different P. cuspidatum tissues clearly demonstrated that the roots and rhizomes generally contained the greatest amounts of stilbenes and anthraquinones compared with other tissues, while the flowers and leaves contained more flavonoids. In addition, 13 compounds were successfully identified, screened and quantified as potential chemical markers for the discrimination of different P. cuspidatum tissues. This study provides chemical bases for the distinct usage of different tissues of P. cuspidatum and could offer some guidance for the further exploitation of this species. 
Supplementary Materials: The following are available online. Figure S1: Typical LC-MS extracted ion chromatograms of metabolites that extracted from different tissues.

Author Contributions: Conceptualization, H.W. and T.C.; methodology, Z.W. and X.W.; software, Z.W., M.C. and X.W.; validation, Z.W., and H.H.; formal analysis, X.W.; investigation, Z.W. and X.W.; resources, H.W.; data curation, H.H. and Z.W.; writing-original draft preparation, Z.W.; writing-review and editing, H.W.; visualization, J.C. and M.C.; supervision, H.W. and T.C.; project administration, H.W. and T.C.; funding acquisition, H.W.

Funding: This work w supported by the National Natural Science Foundation of China (Grant No. 61672489; 61379081) and the Chinese Academy of Sciences (Grant No. KJRH2016).

Acknowledgments: We would like to thank Zhensheng Xie, Lang Sun (Institute of Biophysics, CAS) and Jinging Cao (China Agricultural University) for their help in mass spectrometry data analysis and suggestions about metabolomics.

Conflicts of Interest: The authors declare no conflict of interest.

\section{References}

1. Peng, W.; Qin, R.; Li, X.; Zhou, H. Botany, phytochemistry, pharmacology, and potential application of Polygonum cuspidatum Sieb.et Zucc.: A review. J. Ethnopharmacol. 2013, 148, 729-745. [CrossRef] [PubMed]

2. Fu, J.; Wang, M.; Guo, H.; Tian, Y.; Zhang, Z.; Song, R. Profiling of components of rhizoma et radix polygoni cuspidati by high-performance liquid chromatography with ultraviolet diode-array detector and ion trap/time-of-flight mass spectrometric detection. Pharmacogn. Mag. 2015, 11, 486-501. [PubMed]

3. Zhang, H.; Li, C.; Kwok, S.T.; Zhang, Q.W.; Chan, S.W. A Review of the Pharmacological Effects of the Dried Root of Polygonum cuspidatum (Hu Zhang) and Its Constituents. Evid. Based Complement. Altern. Med. 2013, 2013, 1-13.

4. Zhang, H.; Guo, Y.W.; Yang, Y.D.; Ma, Y.J.; Liu, B.-Y.; Ye, H.-C.; Wang, H.; Ma, L.-Q. Cloning of a novel type III polyketide synthase encoded by a three-intron gene from Polygonum cuspidatum. J. Plant Biochem. Biotechnol. 2012, 23, 104-111. [CrossRef]

5. Xu, M.; Jin, Z.; Ohm, J.-B.; Schwarz, P.; Rao, J.; Chen, B. Improvement of the Antioxidative Activity of Soluble Phenolic Compounds in Chickpea by Germination. J. Agric. Food Chem. 2018, 66, 6179-6187. [CrossRef] [PubMed]

6. Valente, I.M.; Maia, M.R.G.; Malushi, N.; Oliveira, H.M.; Papa, L.; Rodrigues, J.A.; Fonseca, A.J.M.; Cabrita, A.R.J. Profiling of phenolic compounds and antioxidant properties of European varieties and cultivars of Vicia faba L. pods. Phytochemistry 2018, 152, 223-229. [CrossRef] [PubMed]

7. Jayatilake, G.S.; Jayasuriya, H.; Lee, E.S.; Koonchanok, N.M.; Geahlen, R.L.; Ashendel, C.L.; McLaughlin, J.L.; Chang, C.J. Kinase Inhibitors from Polygonum cuspidatum. J. Nat. Prod. 1993, 56, 1805-1810. [CrossRef] [PubMed]

8. Dong, X.; Fu, J.; Yin, X.B.; Cao, S.L.; Li, X.C.; Lin, L.F.; Huyiligeqi Ni, J. Emodin: A Review of its Pharmacology, Toxicity and Pharmacokinetics. Phytother. Res. 2016, 30, 1207-1218. [CrossRef]

9. Editorial Committee of Chinese Pharmacopoeia. Chinese Pharmacopoeia; Editorial Committee of Chinese Pharmacopoeia: Beijing, China, 2015; Volume 1, p. 208.

10. Ma, Y. The Resources, Quality and Pharmaceutical Efficacy of Polygonum cuspidatum Sieb. et Zucc.; Chendu Unversity of TCM: Chendu, China, 2006.

11. Ma, P. Pharmacognostic Studies of Polygonum cuspidatum Sieb. et Zucc. (Polygonaceae); Union Medical College: Beijing, China, 2013.

12. Lin, S.P.; Chu, P.M.; Tsai, S.Y.; Wu, M.H.; Hou, Y.C. Pharmacokinetics and tissue distribution of resveratrol, emodin and their metabolites after intake of Polygonum cuspidatum in rats. J. Ethnopharmacol. 2012, 144, 671-676. [CrossRef]

13. Dong, J.; Wang, H.; Wan, L.; Yuki, H.; Chen, S. Identification and determination of major constituents in Polygonum cuspidatum Sieb. et Zucc. by high performance liquid chromatography/electrospray ionization-ion trap-time-of-flight mass spectrometry. Chin. J. Chromatagr. 2009, 27, 425-430.

14. Kim, Y.S.; Hwang, C.S.; Shin, D.H. Volatile constituents from the leaves of Polygonum cuspidatum S. et Z. and their anti-bacterial activities. Food Microbiol. 2005, 22, 139-144. [CrossRef] 
15. Ding, M.Y.; Jiang, Y.; Yu, X.A.; Zhang, D.; Li, J.; Wang, H.; Shen, J.Y.; Gao, X.M.; Chang, Y.X. Screening of Combinatorial Quality Markers for Natural Products by Metabolomics Coupled With Chemometrics. A Case Study on Pollen Typhae. Front. Pharmacol. 2018, 9, 691. [CrossRef] [PubMed]

16. Geng, Z.; Liu, Y.F.; Gou, Y.; Zhou, Q.M.; He, C.J.; Guo, L.; Zhou, J.; Xiong, L. Metabolomics Study of Cultivated Bulbus Fritillariae Cirrhosae at Different Growth Stages using UHPLC-QTOF-MS Coupled with Multivariate Data Analysis. Phytochem. Anal. 2018, 29, 290-299. [CrossRef] [PubMed]

17. Mais, E.; Alolga, R.N.; Wang, S.L.; Linus, L.O.; Yin, X.J.; Qi, L.W. A comparative UPLC-Q/TOF-MS-based metabolomics approach for distinguishing Zingiber officinale Roscoe of two geographical origins. Food Chem. 2018, 240, 239-244. [CrossRef] [PubMed]

18. Liu, Y.; Li, L.; Xiao, Y.-Q.; Yao, J.-Q.; Li, P.-Y.; Yu, D.-R.; Ma, Y.-L. Global metabolite profiling and diagnostic ion filtering strategy by LC-QTOF MS for rapid identification of raw and processed pieces of Rheum palmatum L. Food Chem. 2016, 192, 531-540. [CrossRef] [PubMed]

19. Chang, J.B.; Lane, M.E.; Yang, M.; Heinrich, M. A Hexa-Herbal TCM Decoction Used to Treat Skin Inflammation: An LC-MS-Based Phytochemical Analysis. Planta Med. 2016, 82, 1134-1141. [CrossRef]

20. Malongane, F.; McGaw, L.J.; Nyoni, H.; Mudau, F.N. Metabolic profiling of four South African herbal teas using high resolution liquid chromatography-mass spectrometry and nuclear magnetic resonance. Food Chem. 2018, 257, 90-100. [CrossRef]

21. Wang, H.; Feng, F. Identification of components in Zhi-Zi-Da-Huang decoction by HPLC coupled with electrospray ionization tandem mass spectrometry, photodiode array and fluorescence detectors. J. Pharm. Biomed. Anal. 2009, 49, 1157-1165. [CrossRef]

22. Chen, S.; Lin, J.; Liu, H.; Gong, Z.; Wang, X.; Li, M.; Aharoni, A.; Yang, Z.; Yu, X. Insights into Tissue-specific Specialized Metabolism in Tieguanyin Tea Cultivar by Untargeted Metabolomics. Molecules 2018, $23,1817$. [CrossRef]

23. Wang, W.X.; Zhang, S.W.; Lv, L.S.; Sang, S.M. A new method to prepare and redefine black tea thearubigins. J. Chromatogr. A 2018, 1563, 82-88. [CrossRef]

24. Li, J.; He, M.; Feng, Y.; Li, T.; Li, Y.; Wu, B.; Ouyang, H.; Yang, S. In-vivo Studies on Metabolism of Luteolin and Luteoloside in Rats. Tradit. Chin. Drug Res. Clin. Plarmacol. 2017, 28, 61-68.

25. Kalogiouri, N.P.; Aalizadeh, R.; Thomaidis, N.S. Application of an advanced and wide scope non-target screening workflow with LC-ESI-QTOF-MS and chemometrics for the classification of the Greek olive oil varieties. Food Chem. 2018, 256, 53-61. [CrossRef]

26. Ji, X.Y.; Liu, X.Q.; Peng, Y.X.; Zhan, R.T.; Xu, H.; Ge, X.J. Comparative analysis of methicillin-sensitive and resistant Staphylococcus aureus exposed to emodin based on proteomic profiling. Biochem. Biophys. Res. Commun. 2017, 494, 318-324. [CrossRef] [PubMed]

27. Fu, J.F.; Wu, S.Y.; Wang, M.; Tian, Y.; Zhang, Z.J.; Song, R. Intestinal metabolism of Polygonum cuspidatum in vitro and in vivo. Biomed. Chromatogr. 2018, 32, e4190. [CrossRef] [PubMed]

28. Vicente, O.; Boscaiu, M. Flavonoids: Antioxidant Compounds for Plant Defence... and for a Healthy Human Diet. Not. Bot. Horti Agrobot. Cluj-Napoca 2018, 46, 14-21. [CrossRef]

29. Liu, S.; Song, F.; Liu, Z. Mass Spectrometry Analysis of Traditional Chinese Medicine, 1st ed.; Science China Press: Beijing, China, 2012.

30. Wu, W.; Liu, Z.Q.; Song, F.R.; Liu, S.Y. Structural analysis of selected characteristic flavones by electrospray tandem mass spectrometry. Anal. Sci. 2004, 20, 1103-1105. [CrossRef]

31. He, M.; Wu, H.; Nie, J.; Yan, P.; Yang, T.B.; Yang, Z.Y.; Pei, R. Accurate recognition and feature qualify for flavonoid extracts from Liang-wai Gan Cao by liquid chromatography-high resolution-mass spectrometry and computational MS/MS fragmentition. J. Pharm. Biomed. Anal. 2017, 146, 37-47. [CrossRef]

32. Mekawy, A.M.M.; Abdelaziz, M.N.; Ueda, A. Apigenin pretreatment enhances growth and salinity tolerance of rice seedlings. Plant Physiol. Biochem. 2018, 130, 94-104. [CrossRef]

33. Karamac, M.; Orak, H.H.; Amarowicz, R.; Orak, A.; Piekoszewski, W. Phenolic contents and antioxidant capacities of wild and cultivated white lupin (Lupinus albus L.) seeds. Food Chem. 2018, 258, 1-7. [CrossRef] [PubMed]

34. Nabavi, S.F.; Braidy, N.; Gortzi, O.; Sobarzo-Sanchez, E.; Daglia, M.; Skalicka-Wozniak, K.; Nabavi, S.M. Luteolin as an anti-inflammatory and neuroprotective agent: A brief review. Brain Res. Bull. 2015, 119, 1-11. [CrossRef] [PubMed] 
35. Kashyap, D.; Sharma, A.; Tuli, H.S.; Sak, K.; Garg, V.K.; Buttar, H.S.; Setzer, W.N.; Sethi, G. Apigenin: A natural bioactive flavone-type molecule with promising therapeutic function. J. Funct. Foods 2018, 48, 457-471. [CrossRef]

36. Wang, T.Y.; Li, Q.; Bi, K.S. Bioactive flavonoids in medicinal plants: Structure, activity and biological fate. Asian J. Pharm. Sci. 2018, 13, 12-23. [CrossRef]

37. Ni, J.; Dong, L.X.; Jiang, Z.F.; Yang, X.L.; Sun, Z.H.; Li, J.X.; Wu, Y.H.; Xu, M.J. Salicylic acid-induced flavonoid accumulation in Ginkgo biloba leaves is dependent on red and far-red light. Ind. Crop. Prod. 2018, 118, 102-110. [CrossRef]

38. Karakaya, S.; Koca, M.; Yılmaz, S.V.; Yıldırım, K.; Pınar, N.M.; Demirci, B.; Brestic, M.; Sytar, O. Molecular Docking Studies of Coumarins Isolated from Extracts and Essential Oils of Zosima absinthifolia Link as Potential Inhibitors for Alzheimer's Disease. Molecules 2019, 24, 722. [CrossRef] [PubMed]

39. Liu, Z.-S.; Cai, H.; Xue, W.; Wang, M.; Xia, T.; Li, W.-J.; Xing, J.-Q.; Zhao, M.; Huang, Y.-J.; Chen, S.; et al. G3BP1 promotes DNA binding and activation of cGAS. Nat. Immunol. 2018, 20, 18-28. [CrossRef] [PubMed]

40. Zhang, L.; Huang, Y.; Lian, M.; Fan, Z.; Tian, Y.; Wang, Y.; Kang, H.; Liu, S.; Liu, S.; Li, T.; et al. Metabolic profiling of hepatitis B virus-related hepatocellular carcinoma with diverse differentiation grades. Oncol. Lett. 2017, 13, 1204-1210. [CrossRef] [PubMed]

41. Nickler, M.; Ottiger, M.; Steuer, C.; Huber, A.; Anderson, J.B.; Mueller, B.; Schuetz, P. Systematic review regarding metabolic profiling for improved pathophysiological understanding of disease and outcome prediction in respiratory infections. Resp. Res. 2015, 16, 125. [CrossRef]

42. Mo, D.; Mingming, S.; Xianfu, G.; Tie, Z.; Aihua, Z.; Guoxiang, X.; Yunping, Q.; Mingmei, Z.; Zhong, L.; Wei, J. Metabolite profiling of Panax notoginseng using UPLC-ESI-MS. Phytochemistry 2008, 69, 2237-2244.

43. Li, P.; AnandhiSenthilkumar, H.; Wu, S.-B.; Liu, B.; Guo, Z.-Y.; Fata, J.E.; Kennelly, E.J.; Long, C.-L. Comparative UPLC-QTOF-MS-based metabolomics and bioactivities analyses of Garcinia oblongifolia. J. Chromatogr. B Anal. Technol. Biomed. Life Sci. 2016, 1011, 179-195. [CrossRef]

44. Benton, H.P.; Ivanisevic, J.; Mahieu, N.G.; Kurczy, M.E.; Johnson, C.H.; Franco, L.; Rinehart, D.; Valentine, E.; Gowda, H.; Ubhi, B.K.; et al. Autonomous Metabolomics for Rapid Metabolite Identification in Global Profiling. Anal. Chem. 2015, 87, 884-891. [CrossRef]

Sample Availability: Samples of the compounds are not available from the authors.

(C) 2019 by the authors. Licensee MDPI, Basel, Switzerland. This article is an open access article distributed under the terms and conditions of the Creative Commons Attribution (CC BY) license (http://creativecommons.org/licenses/by/4.0/). 